\title{
Coeliac Disease: From Triggering Factors to Treatment
}

\author{
Erna Sziksz ${ }^{1,2, *}$, Péter Vörös ${ }^{2}$, Gábor Veres ${ }^{2}$, Andrea Fekete ${ }^{2,3}$, Ádám Vannay ${ }^{1,2}$ \\ ${ }^{1}$ Research Group for Pediatrics and Nephrology, Hungarian Academy of Sciences and Semmelweis University, Budapest, Hungary \\ ${ }^{2}$ First Department of Pediatrics, Semmelweis University, Budapest, Hungary \\ 3“Momentum” Diabetes Research Group, Hungarian Academy of Sciences and Semmelweis University, Budapest, Hungary \\ *Corresponding author: szikszerna@gmail.com
}

Received December 02, 2013; Revised December 08, 2013; Accepted December 12, 2013

\begin{abstract}
Coeliac disease (CD) or gluten sensitive enteropathy is one of the most common inflammatory diseases of the small intestine with estimated prevalence of $1 \%$ in the population. Its incidence is increasing and seems to be higher than expected in the pediatric population associated with unfavorable impact on the quality of life. The aim of the present review is to highlight the main triggering factors leading to the development of $\mathrm{CD}$ and its pathomechanism with a special outlook to the recent therapeutic approaches.
\end{abstract}

Keywords: coeliac disease, triggering factors, pathomechanism, therapy

Cite This Article: Erna Sziksz, Péter Vörös, Gábor Veres, Andrea Fekete, and Ádám Vannay, "Coeliac Disease: From Triggering Factors to Treatment.” International Journal of Celiac Disease 1, no. 1 (2013): 9-13. doi: 10.12691/ijcd-1-1-5.

\section{Introduction}

CD occurs in genetically susceptible individuals triggered by chronic exposure to dietary gluten and related prolamins [1]. Approximately $1 \%$ of the population is affected and its prevalence is increased over the last decades [2,3]. This tendency suggests that beside of the genetic predisposition the environmental stress factors also have significant impact on the development of CD [4]. CD often becomes active after surgery, pregnancy, childbirth, viral infection, emotional or other stress situations [5]. The disease leads to intestinal inflammation, villous atrophy, and crypt hyperplasia of the small intestine. In the pathomechanism of CD both the adaptive and innate immunity may be involved 6 . Presumed disease is mainly detected by serologic screening for the presence of tissue transglutaminase (tTG) specific immunglobulin A antibodies [7]. CD may be associated also with various extra-intestinal complications, including isolated iron deficiency anemia, bone and skin disease, infertility, endocrine and neurologic disorders [8].

In the present review we give an overview about the role of genetic and environmental factors in the development of CD and about the major processes of its pathomechanism including the mucosal immune responses and epithelial barrier damage. Finally, we discuss the main strategies to find alternative therapies for CD.

\subsection{Role of Genetic and Environmental Factors}

The genetic predisposition is associated with the major histocompatibility complex (MHC) region on chromosome 6p21. About $90 \%$ of CD patients carry the human leukocyte antigen (HLA) DQ2 and the remainder expresses DQ8 [9]. Patients with CD who are negative for both DQ2 and DQ8 rarely occurs [10].

One of the most important environmental factors which can initiate the exacerbation of the symptoms of CD is gluten and its related prolamins (hordein, secalin, zein). The presence of gliadin, the alcohol-soluble fraction of gluten that contains the majority of toxic components, may initiate inappropriate innate and adaptive immune response leading to the development of $\mathrm{CD}$.

Gliadins are rich in glutamine and proline, which protect them from proteolytic degradation by intestinal brush-border membrane proteases, they cannot be absorbed and remain in the intestinal lumen after gluten ingestion [11]. Due to intestinal infections or other disturbances in the intestinal permeability the undigested gliadin fractions pass through the epithelial barrier [2]. In the lamina propria tTGs become activated and catalyze polyamination or deamidation at selective glutamine residues converting them to negatively charged glutamate [12]. This modification promotes the binding of gliadinderived fragments to HLA-DQ2 and HLA-DQ8 molecules resulting in antigen presentation and activation of glutenspecific $\mathrm{T}$ cells leading to permanent inflammation and mucosal damage [13].

Beside the most relevant gliadins there are also other environmental factors which play crucial role in the pathogenesis of CD including mechanical, chemical or oxidative stress which may all impair epithelial barrier function and facilitate inflammation [14,15].

\subsection{Mucosal Immune Responses}

Gliadin peptides passing through the damaged epithelial layer induce innate and adaptive immune responses characterized by the infiltration of immune cells in the lamina propria. 
The importance of the adaptive immune response to gluten has been well established. After the presentation of deamidated gluten-derived peptides through HLA DQ2 and DQ8 molecules to CD4+ T helper cells further T cells and B lymphocytes become activated which produce antigluten and anti-tTG2 antibodies in the lamina propria [13]. Activated T cells express proinflammatory cytokines, e.g. tumor necrosis factor alpha (TNF $\alpha$ ) and interferon gamma (IFN $\gamma$ ) triggering the release of matrix metalloproteinases by the local fibroblasts causing epithelial cell damage and tissue remodeling, respectively [16].

Beside the involvement of the components of adaptive immunity the central role of innate immune system has been also suggested in the pathogenesis of CD [6].

Recently increased expression of pattern recognition receptors including Toll-like receptor (TLR) 2 and 4, the well known participants of the innate immune response, was demonstrated in the duodenal mucosa of children with CD [17]. Increased expression of molecular chaperons, heat shock proteins (HSPs) were also demonstrated in patients with CD $[18,19]$. It is hypothesized that HSPs as ligands for TLRs induce antigen presentation process and serve as "danger signal" for the immune system [20]. Activation of TLRs on the surface of macrophages and dendritic cells leads to the upregulation of MHC and costimulatory molecules and enhanced expression of proinflammatory cytokines and chemokines [21].

Gliadin-induced innate immune response is also characterized by the increased expression of interleukin15 produced mainly by enterocytes, which activates intraepithelial CD8+ (cytotoxic) T lymphocytes leading to the destruction of the epithelial cells thus contributing to the damage of the barrier function.

\subsection{Mucosal Barrier Damage}

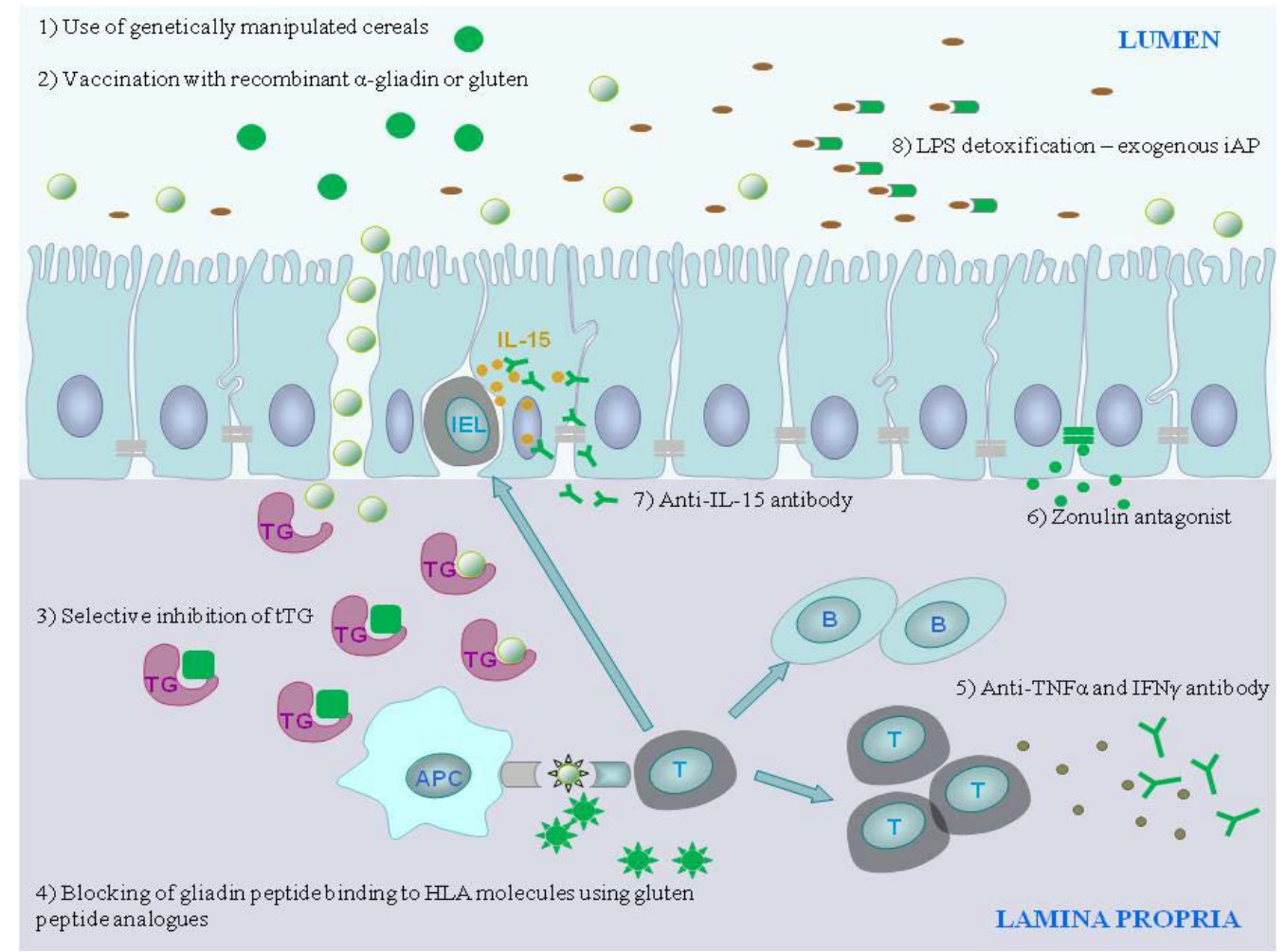

Figure 1. Alternative therapeutic strategies targeting the pathomechanism of CD. Abbreviations: tTG: tissue transglutaminase; IEL: intraepithelial lymphocytes; IL-15: interleukin-15; APC: antigen presenting cell; HLA: human leukocyte antigen, T: T lymphocyte; B: B lymphocyte; TNF: tumor necrosis factor; IFN: interferon, LPS: lipopolysaccharide 


\subsection{Therapy}

Since undigested gluten and related prolamins are the key triggering factors of $\mathrm{CD}$, it is not surprising that all of the therapeutic interventions aim to eliminate gluten or attenuate its biological effects. At present gluten free diet (GFD) is the accepted treatment for $\mathrm{CD}$, which involves the lifelong elimination of wheat, rye, barley and other gluten-containing foods from the diet. The strict GFD is costly and has an adverse impact on the quality of life [32]. In addition GFD is not fully effective in a significant part of the patients who are unresponsive [4], therefore new therapeutic strategies are under development [33] (Figure 1).

As an alternative therapeutic approach of CD, the use of genetically altered wheat strains lacking harmful gluten epitopes [34] and the detoxification of dietary gluten by enzymatic degradation of gliadin fragments [35] came into the focus and are in preclinical stage. Other therapeutic strategies aim to induce mucosal tolerance by intranasal administration of recombinant $\alpha$-gliadin, which resulted in the downregulation of the immune response against whole gliadin [36]. Furthermore, immunization with gluten vaccines is under clinical testing showing a well tolerance [37].

Other therapeutic interventions are directed to modulate the immune responses against gliadin peptides and to inhibit the development of inflammation. The blocking of the antigen presentation process through the selective inhibition of tTG2 (e.g. use of cystamine, imidazolium derivates, dihydroisoaxole compounds) are only partly effective because of the risk of undesired interactions with vital biological pathways, thus clinical trials were not undertaken [33, 38]. However, the inhibition of antigen presentation through the blocking of gliadin peptide binding to HLA molecules using gluten peptide analogues is a more effective potential treatment for CD [39]. Likewise the prevention of the recruitment of lymphocytes targeting the chemokine receptor and ligand interactions e.g. CCR9 and CCL25 [40] and CXCR3 and CXCL10 [41] and the modulation of inflammation by monoclonal antibodies against TNF- $\alpha$ (Infliximab) [42] and IFN- $\gamma$ [43] are another attractive therapeutic options for CD [44].

Another way of action is the preservation of the structure of intestinal barrier: the modulation of the intestinal permeability by zonulin antagonist AT-1001 is a completed phase IIb complementary approach with promising results [45]. Also the administration of a specific anti-IL-15 antibody was able to reverse the intestinal barrier damage through the induction of apoptosis of intraepithelial lymphocytes [46], however clinical studies for CD have been not yet performed. As another strategy to maintain the integrity of the epithelial layer may be the administration of exogenous intestinal alkaline phosphatase (iAP), an enzyme with advantageous bacterial LPS detoxification capacity [47], since its decreased level was reported in CD [48] and when added to newborns with necrotizing enterocolitis significant decrease in inflammatory cytokine expression and intestinal injury were observed [49].

\section{Conclusions}

$\mathrm{CD}$ is a genetically determined autoimmune disease triggered by dietary gluten and related prolamins. The worldwide prevalence of $\mathrm{CD}$ is $1 \%$ and is still continuously increasing. At present the accepted treatment of $\mathrm{CD}$ is a strict and lifelong elimination of gluten from the diet. This therapy is circumstantial and negatively influences the quality of life of both the affected individuals and also their families. In a significant part of the patients GFD is ineffective indicating the need for other alternative therapies. Accordingly different therapies are under development aiming the alternative elimination of gluten from the nutrition or the modulation of the immune responses against gluten.

\section{Acknowledgments}

This work was supported by grants OTKANK84087/2010, -K105530, -PD83431,“Lendület” Research Grant LP2011-008, and KMR_12-1-2012-0074. Vannay Á is a holder of the János Bolyai Research grant by János Bolyai Research Scholarschip of the Hungarian Academy of Sciences.

\section{Statement of Competing Interests}

The authors have no competing interests.

\section{List of Abbreviations}

\author{
CD: coeliac disease \\ tTG: tissue transglutaminase \\ MHC: major histocompatibility complex \\ HLA: human leukocyte antigen \\ TNFa: tumor necrosis factor alpha \\ IFN $\gamma$ : interferon gamma \\ TLR: toll-like receptor \\ HSP: heat shock proteins \\ HIF: hypoxia-inducible factor \\ PARK7: Parkinson's disease 7 \\ ROS: reactive oxygen species \\ GFD: gluten free diet \\ iAP: intestinal alkaline phosphatase
}

\section{References}

[1] Setty, M., Hormaza, L., Guandalini, S. Celiac disease: risk assessment, diagnosis, and monitoring. Molecular diagnosis \& therapy, 12 (5):289-98. 2008.

[2] Green, P.H., Cellier, C. Celiac disease. The New England journal of medicine, 357 (17):1731-43. 2007.

[3] Makovicky, P., Rimarova, K., Boor, A., Makovicky, P., Vodicka, P., Samasca, G., Kruzliak, P. Correlation between antibodies and histology in celiac disease: incidence of celiac disease is higher than expected in the pediatric population. Molecular medicine reports, 8 (4):1079-83. 2013.

[4] Rubio-Tapia, A., Murray, J.A. Classification and management of refractory coeliac disease. Gut, 59 (4):547-57. 2010

[5] Baldassarre, M., Laneve, A.M., Grosso, R., Laforgia, N. Celiac disease: pathogenesis and novel therapeutic strategies. Endocrine, metabolic \& immune disorders drug targets, 8 (3):152-8. 2008.

[6] Jabri, B., Sollid, L.M. Mechanisms of disease: immunopathogenesis of celiac disease. Nature clinical practice. Gastroenterology \& hepatology, 3 (9): 516-25. 2006.

[7] Rostom, A., Dube, C., Cranney, A., Saloojee, N., Sy, R., Garritty, C., Sampson, M., Zhang, L., Yazdi, F., Mamaladze, V., Pan, I., MacNeil, J., Mack, D., Patel, D., Moher, D. The diagnostic accuracy of serologic tests for celiac disease: a systematic review. Gastroenterology, 128 (4 Suppl 1):S38-46. 2005. 
[8] Lauret, E., Rodrigo, L. Celiac disease and autoimmune-associated conditions. BioMed research international, 2013:127589. 2013.

[9] Schuppan, D., Dennis, M.D., Kelly, C.P. Celiac disease: epidemiology, pathogenesis, diagnosis, and nutritional management. Nutrition in clinical care : an official publication of Tufts University, 8 (2):54-69. 2005.

[10] Karell, K., Louka, A.S., Moodie, S.J., Ascher, H., Clot, F., Greco, L., Ciclitira, P.J., Sollid, L.M., Partanen, J., European Genetics Cluster on Celiac, D. HLA types in celiac disease patients not carrying the DQA1*05-DQB1*02 (DQ2) heterodimer: results from the European Genetics Cluster on Celiac Disease. Human immunology, 64 (4):469-77. 2003.

[11] Shan, L., Molberg, O., Parrot, I., Hausch, F., Filiz, F., Gray, G.M., Sollid, L.M., Khosla, C. Structural basis for gluten intolerance in celiac sprue. Science, 297 (5590):2275-9. 2002

[12] Caccamo, D., Curro, M., Ientile, R. Potential of transglutaminase 2 as a therapeutic target. Expert opinion on therapeutic targets, 14 (9):989-1003. 2010.

[13] Sollid, L.M. Coeliac disease: dissecting a complex inflammatory disorder. Nature reviews. Immunology, 2 (9):647-55. 2002.

[14] Lewis, K., McKay, D.M. Metabolic stress evokes decreases in epithelial barrier function. Annals of the New York Academy of Sciences, 1165:327-37. 2009.

[15] Szaflarska-Poplawska, A., Siomek, A., Czerwionka-Szaflarska, M., Gackowski, D., Rozalski, R., Guz, J., Szpila, A., Zarakowska, E., Olinski, R. Oxidatively damaged DNA/oxidative stress in children with celiac disease. Cancer epidemiology, biomarkers \& prevention : a publication of the American Association for Cancer Research, cosponsored by the American Society of Preventive Oncology, 19 (8):1960-5. 2010.

[16] Schuppan, D., Junker, Y., Barisani, D. Celiac disease: from pathogenesis to novel therapies. Gastroenterology, 137 (6):191233. 2009.

[17] Szebeni, B., Veres, G., Dezsofi, A., Rusai, K., Vannay, A., Bokodi, G., Vasarhelyi, B., Korponay-Szabo, I.R., Tulassay, T., Arato, A. Increased mucosal expression of Toll-like receptor (TLR)2 and TLR4 in coeliac disease. Journal of pediatric gastroenterology and nutrition, 45 (2):187-93. 2007.

[18] Iltanen, S., Rantala, I., Laippala, P., Holm, K., Partanen, J., Maki, M. Expression of HSP-65 in jejunal epithelial cells in patients clinically suspected of coeliac disease. Autoimmunity, 31 (2):12532. 1999.

[19] Sziksz, E., Veres, G., Vannay, A., Prokai, A., Gal, K., Onody, A., Korponay-Szabo, I.R., Reusz, G., Szabo, A., Tulassay, T., Arato, A., Szebeni, B. Increased heat shock protein 72 expression in celiac disease. Journal of pediatric gastroenterology and nutrition, 51 (5):573-8. 2010.

[20] Li, Z., Menoret, A., Srivastava, P. Roles of heat-shock proteins in antigen presentation and cross-presentation. Current opinion in immunology, 14 (1):45-51. 2002.

[21] Takeda, K., Kaisho, T., Akira, S. Toll-like receptors. Annual review of immunology, 21:335-76. 2003.

[22] Jeon, M.K., Klaus, C., Kaemmerer, E., Gassler, N. Intestinal barrier: Molecular pathways and modifiers. World journal of gastrointestinal pathophysiology, 4 (4):94-99. 2013.

[23] Laukoetter, M.G., Bruewer, M., Nusrat, A. Regulation of the intestinal epithelial barrier by the apical junctional complex. Current opinion in gastroenterology, 22 (2):85-9. 2006.

[24] Fasano, A. Zonulin, regulation of tight junctions, and autoimmune diseases. Annals of the New York Academy of Sciences, 1258:2533. 2012.

[25] Tripathi, A., Lammers, K.M., Goldblum, S., Shea-Donohue, T., Netzel-Arnett, S., Buzza, M.S., Antalis, T.M., Vogel, S.N., Zhao, A., Yang, S., Arrietta, M.C., Meddings, J.B., Fasano, A. Identification of human zonulin, a physiological modulator of tight junctions, as prehaptoglobin-2. Proceedings of the National Academy of Sciences of the United States of America, 106 (39):16799-804. 2009.

[26] Catalioto, R.M., Maggi, C.A., Giuliani, S. Intestinal epithelial barrier dysfunction in disease and possible therapeutical interventions. Current medicinal chemistry, 18 (3):398-426. 2011.

[27] Niecknig, H., Tug, S., Reyes, B.D., Kirsch, M., Fandrey, J., Berchner-Pfannschmidt, U. Role of reactive oxygen species in the regulation of HIF-1 by prolyl hydroxylase 2 under mild hypoxia. Free radical research, 46 (6):705-17. 2012.

[28] Diosdado, B., van Oort, E., Wijmenga, C. "Coelionomics": towards understanding the molecular pathology of coeliac disease.
Clinical chemistry and laboratory medicine: CCLM / FESCC, 43 (7):685-95. 2005.

[29] Vasseur, S., Afzal, S., Tardivel-Lacombe, J., Park, D.S., Iovanna, J.L., Mak, T.W. DJ-1/PARK7 is an important mediator of hypoxia-induced cellular responses. Proceedings of the National Academy of Sciences of the United States of America, 106 (4):1111-6. 2009.

[30] Voros, P., Sziksz, E., Himer, L., Onody, A., Pap, D., Frivolt, K., Szebeni, B., Lippai, R., Gyorffy, H., Fekete, A., Brandt, F., Molnar, K., Veres, G., Arato, A., Tulassay, T., Vannay, A. Expression of PARK7 is increased in celiac disease. Virchows Archiv: an international journal of pathology, 463 (3):401-8. 2013.

[31] Vannay, A., Sziksz, E., Prokai, A., Veres, G., Molnar, K., Szakal, D.N., Onody, A., Korponay-Szabo, I.R., Szabo, A., Tulassay, T., Arato, A., Szebeni, B. Increased expression of hypoxia-inducible factor 1alpha in coeliac disease. Pediatric research, 68 (2):118-22. 2010.

[32] Lee, A., Newman, J.M. Celiac diet: its impact on quality of life. Journal of the American Dietetic Association, 103 (11):1533-5. 2003.

[33] Rashtak, S., Murray, J.A. Review article: coeliac disease, new approaches to therapy. Alimentary pharmacology \& therapeutics, 35 (7):768-81. 2012.

[34] van den Broeck, H.C., van Herpen, T.W., Schuit, C., Salentijn, E.M., Dekking, L., Bosch, D., Hamer, R.J., Smulders, M.J., Gilissen, L.J., van der Meer, I.M. Removing celiac disease-related gluten proteins from bread wheat while retaining technological properties: a study with Chinese Spring deletion lines. BMC plant biology, 9:41. 2009.

[35] Gass, J., Bethune, M.T., Siegel, M., Spencer, A., Khosla, C. Combination enzyme therapy for gastric digestion of dietary gluten in patients with celiac sprue. Gastroenterology, 133 (2):472-80. 2007.

[36] Senger, S., Luongo, D., Maurano, F., Mazzeo, M.F., Siciliano, R.A., Gianfrani, C., David, C., Troncone, R., Auricchio, S., Rossi, M. Intranasal administration of a recombinant alpha-gliadin downregulates the immune response to wheat gliadin in DQ8 transgenic mice. Immunology letters, 88 (2):127-34. 2003.

[37] Aziz, I., Evans, K.E., Papageorgiou, V., Sanders, D.S. Are patients with coeliac disease seeking alternative therapies to a gluten-free diet? Journal of gastrointestinal and liver diseases: JGLD, 20 (1):27-31. 2011.

[38] Maiuri, L., Ciacci, C., Ricciardelli, I., Vacca, L., Raia, V., Rispo, A., Griffin, M., Issekutz, T., Quaratino, S., Londei, M. Unexpected role of surface transglutaminase type II in celiac disease. Gastroenterology, 129 (5):1400-13. 2005.

[39] Xia, J., Bergseng, E., Fleckenstein, B., Siegel, M., Kim, C.Y., Khosla, C., Sollid, L.M. Cyclic and dimeric gluten peptide analogues inhibiting DQ2-mediated antigen presentation in celiac disease. Bioorganic \& medicinal chemistry, 15 (20):6565-73. 2007

[40] Rivera-Nieves, J., Ho, J., Bamias, G., Ivashkina, N., Ley, K., Oppermann, M., Cominelli, F. Antibody blockade of CCL25/CCR9 ameliorates early but not late chronic murine ileitis. Gastroenterology, 131 (5):1518-29. 2006.

[41] Booth, V., Keizer, D.W., Kamphuis, M.B., Clark-Lewis, I., Sykes, B.D. The CXCR3 binding chemokine IP-10/CXCL10: structure and receptor interactions. Biochemistry, 41 (33):10418-25. 2002.

[42] Gillett, H.R., Arnott, I.D., McIntyre, M., Campbell, S., Dahele, A., Priest, M., Jackson, R., Ghosh, S. Successful infliximab treatment for steroid-refractory celiac disease: a case report. Gastroenterology, 122 (3):800-5. 2002.

[43] Bethune, M.T., Siegel, M., Howles-Banerji, S., Khosla, C. Interferon-gamma released by gluten-stimulated celiac diseasespecific intestinal $\mathrm{T}$ cells enhances the transepithelial flux of gluten peptides. The Journal of pharmacology and experimental therapeutics, 329 (2):657-68. 2009.

[44] Anderson, R.P., van Heel, D.A., Tye-Din, J.A., Jewell, D.P., Hill, A.V. Antagonists and non-toxic variants of the dominant wheat gliadin T cell epitope in coeliac disease. Gut, 55 (4):485-91. 2006.

[45] Paterson, B.M., Lammers, K.M., Arrieta, M.C., Fasano, A., Meddings, J.B. The safety, tolerance, pharmacokinetic and pharmacodynamic effects of single doses of AT-1001 in coeliac disease subjects: a proof of concept study. Alimentary pharmacology \& therapeutics, 26 (5):757-66. 2007.

[46] Malamut, G., El Machhour, R., Montcuquet, N., Martin-Lanneree, S., Dusanter-Fourt, I., Verkarre, V., Mention, J.J., Rahmi, G., Kiyono, H., Butz, E.A., Brousse, N., Cellier, C., Cerf-Bensussan, N., Meresse, B. IL-15 triggers an antiapoptotic pathway in human 
intraepithelial lymphocytes that is a potential new target in celiac disease-associated inflammation and lymphomagenesis. The Journal of clinical investigation, 120 (6):2131-43. 2010.

[47] Goldberg, R.F., Austen, W.G., Jr., Zhang, X., Munene, G., Mostafa, G., Biswas, S., McCormack, M., Eberlin, K.R., Nguyen, J.T., Tatlidede, H.S., Warren, H.S., Narisawa, S., Millan, J.L., Hodin, R.A. Intestinal alkaline phosphatase is a gut mucosal defense factor maintained by enteral nutrition. Proceedings of the National Academy of Sciences of the United States of America, 105 (9):3551-6. 2008.
[48] Molnar, K., Vannay, A., Sziksz, E., Banki, N.F., Gyorffy, H., Arato, A., Dezsofi, A., Veres, G. Decreased mucosal expression of intestinal alkaline phosphatase in children with coeliac disease. Virchows Archiv: an international journal of pathology, 460 (2):157-61. 2012.

[49] Rentea, R.M., Liedel, J.L., Fredrich, K., Welak, S.R., Pritchard, K.A., Jr., Oldham, K.T., Simpson, P.M., Gourlay, D.M. Intestinal alkaline phosphatase administration in newborns decreases systemic inflammatory cytokine expression in a neonatal necrotizing enterocolitis rat model. The Journal of surgical research, 177 (2):228-34. 2012. 